\title{
Flat bands and entanglement in the Kitaev ladder
}

\author{
Ritu Nehra, ${ }^{*}$ Devendra Singh Bhakuni, ${ }^{\dagger}$ Ajith Ramachandran,,${ }^{\ddagger}$ and Auditya Sharma ${ }^{\S}$ \\ Department of Physics, Indian Institute of Science Education and Research, Bhopal 462066, India
}

(Received 4 November 2019; accepted 6 February 2020; published 20 February 2020)

\begin{abstract}
We report the existence of flat bands in a p-wave superconducting Kitaev ladder. We identify two sets of parameters for which the Kitaev ladder sustains flat bands. These flat bands are accompanied by highly localized eigenstates known as compact localized states. Invoking a Bogoliubov transformation, the Kitaev ladder can be mapped into an interlinked cross-stitch lattice. The mapping helps to reveal the compactness of the eigenstates, each of which covers only two unit cells of the interlinked cross-stitch lattice. The Kitaev Hamiltonian undergoes a topological-to-trivial phase transition when certain parameters are fine tuned. Correlation matrix techniques allow us to compute entanglement entropy of the many-body eigenstates. The study of entanglement entropy affords fresh insight into the topological phase transitions in the model. Sharp features in entanglement entropy when bands cross indicate a deep underlying relationship between entanglement entropy and dispersion.
\end{abstract}

DOI: 10.1103/PhysRevResearch.2.013175

\section{INTRODUCTION}

In the last few years, dispersionless bands, also known as flat bands, have received a great deal of attention within the condensed matter physics community [1-3]. In tightbinding systems which have periodic lattice potentials, the Bloch theorem [4] ensures the existence of a well-defined band structure for a single particle which, in turn, uniquely defines the group velocity of the particle. Certain carefully tuned lattice Hamiltonians may exhibit one or more flat bands in the band structure, where the energy is independent of momentum, resulting in zero group velocity. The study of the suppression of wave transport induced by flat bands has been carried out in a number of works $[2,5,6]$. Since the wave equations which govern the dynamics can be generalized, flat band physics has been explored in diverse systems ranging from Hubbard models $[7,8]$ to photonic crystals [9-11] and Bose-Einstein condensates [12,13].

The eigenstates corresponding to flat bands are highly localized, covering a finite number of lattice sites, and are therefore referred to as compact localized states (CLS) [2]. One of the prime strategies for finding flat band systems has been to first identify CLS, and then engineer Hamiltonians that could support them. A number of recent studies [5,14,15] have searched for flat band generating algorithms with the aim of identifying more and more flat band networks. Many such studies have been based on noninteracting particles, and focus on geometries which support a flat band through destructive

\footnotetext{
*ritunehra@iiserb.ac.in

†devendra123@iiserb.ac.in

¥jithr@iiserb.ac.in

§auditya@iiserb.ac.in
}

Published by the American Physical Society under the terms of the Creative Commons Attribution 4.0 International license. Further distribution of this work must maintain attribution to the author(s) and the published article's title, journal citation, and DOI. interference properties of the eigenstates $[14,16,17]$. In this work, we report the existence of flat bands in a rather simple superconducting Kitaev ladder Hamiltonian system, which has hitherto been unexplored in this context. Subjecting the Kitaev ladder to a Bogoliubov transformation allows us to map it to an interlinked cross-stitch lattice and thus obtain the explicit compact localized states in the transformed basis. One key aspect of our work is that despite the very simple geometry of the lattice, a flat band becomes possible due to the $p$-wave superconducting term. Our tests indicate that the introduction of a pairing term to generate flat bands may well be quite general beyond the specifics of the current model, and could potentially open up a new avenue for flat-band engineering.

The Kitaev ladder [18-22] is a generalization of the Kitaev chain [23], which is a canonical system that admits a topological phase transition [24]. The simultaneous presence of both the superconducting term and the ladder geometry leads to many rich phenomena including some exotic transport properties [25]. Furthermore, this system exhibits multiple topologically nontrivial phases characterized by different symmetry classes, which may be accessed by tuning different parameters. Quantum entanglement [6,26-29] has emerged as a key tool of study whenever quantum phase transitions are present. This includes topological-to-trivial phase transitions [30-32], Meissner-to-vortex phase transitions [27,33], localization/delocalization transitions [34,35], and many others [36-39]. In this paper, we study entanglement in the manybody eigenstates of the Kitaev ladder. Since the computation of entanglement in the eigenstates of systems that feature a superconducting term is challenging, the literature [40] on this subject is rather sparse, and our work is to be seen as a contribution to filling this void. Thus, our paper is a study of the interplay of flat bands, topological properties, and entanglement properties of the Kitaev ladder.

The layout of the paper is as follows: We start by introducing the Hamiltonian for the two-leg Kitaev ladder. The conditions for the flat bands and a discussion of compact localized states are provided in the subsequent section. The 
(a)
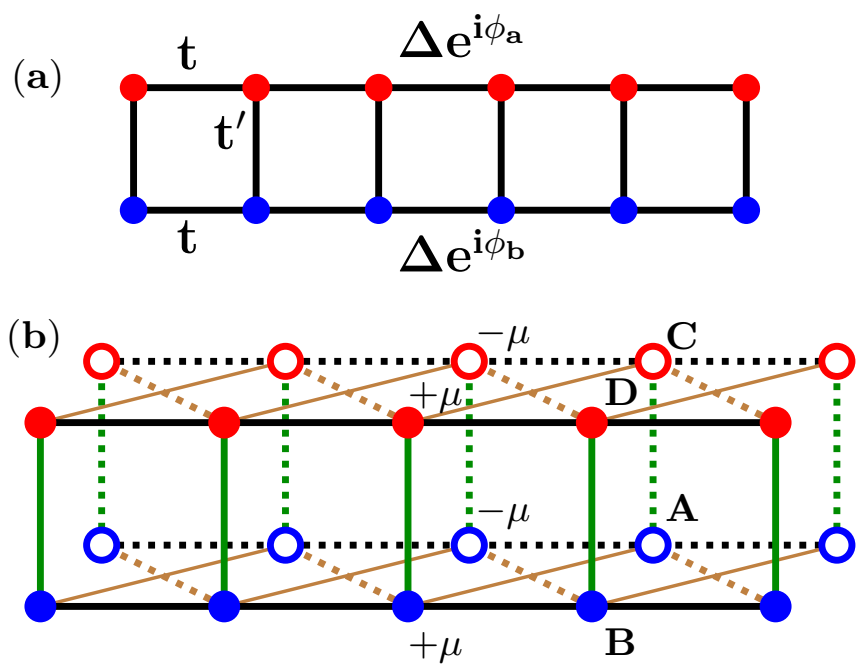

FIG. 1. Schematic representations of (a) the Kitaev ladder, and (b) interlinked cross-stitch lattice. The solid (dotted) lines show positive (negative) hopping. The open and closed circles represent negative $(-\mu)$ and positive $(\mu)$ onsite energies, respectively. The intraleg hoppping $t$ is shown by black lines, interleg hopping $t^{\prime}$ is shown using green lines, and brown color is used for superconducting coupling $\Delta$. The red color circles represent the upper leg of the ladder, i.e., $\sigma=1$ and blue circles represent $\sigma=2$. Using a Bogoliubov transformation, the Kitaev chain Hamiltonian can be mapped to the interlinked cross-stitch lattice Hamiltonian.

next section discusses the topological properties of the system before moving on to a study of entanglement entropy. Finally, we summarize our main findings in the last section.

\section{KITAEV LADDER}

The Kitaev ladder [23,41] [Fig. 1(a)] consists of two Kitaev chains connected to one another through interleg hopping. This system is described by the tight-binding Hamiltonian

$$
\begin{aligned}
H= & -t \sum_{\substack{n \\
\sigma=1,2}} c_{n+1, \sigma}^{\dagger} c_{n, \sigma}-\mu \sum_{\substack{n \\
\sigma=1,2}} c_{n, \sigma}^{\dagger} c_{n, \sigma}-t^{\prime} \sum_{n} c_{n, 1}^{\dagger} c_{n, 2} \\
& -\Delta \sum_{\substack{n \\
\sigma=1,2}} e^{i \phi_{\sigma}} c_{n+1, \sigma}^{\dagger} c_{n, \sigma}^{\dagger}+\text { H.c. },
\end{aligned}
$$

where $t$ is the intraleg hopping amplitude, $c_{n, \sigma}^{\dagger}\left(c_{n, \sigma}\right)$ are creation (annihilation) operators on the $n$th site of the ladder with $\sigma=1,2$ running over two legs of the ladder, and $\mu$ is the onsite chemical potential. The interleg hopping on the ladder is $t^{\prime}$ and the superconducting gap is $\Delta$ with a phase factor $e^{i \phi_{\sigma}}$ in each leg of the ladder. As the annihilation of an electron is equivalent to the creation of a hole, one can write $d_{n, \sigma}^{\dagger}=c_{n, \sigma}, d_{n, \sigma}=c_{n, \sigma}^{\dagger}$, and the Hamiltonian can be redefined in momentum space as $[25,41]$

$$
H=\sum_{k} \Gamma_{k}^{\dagger} \mathcal{H}(k) \Gamma_{k}
$$

where

$$
\Gamma_{k}^{\dagger}=\left[\begin{array}{llll}
c_{k, 1}^{\dagger} & d_{k, 1}^{\dagger} & c_{k, 2}^{\dagger} & d_{k, 2}^{\dagger}
\end{array}\right], \quad \Gamma_{k}=\left[\begin{array}{c}
c_{k, 1} \\
d_{k, 1} \\
c_{k, 2} \\
d_{k, 2}
\end{array}\right] .
$$

Thus, the overall Hamiltonian in $k$ space can be given as

$$
\mathcal{H}(k)=\left[\begin{array}{cccc}
-\epsilon_{\mu, k} & t_{\Delta, \phi_{1}} & -t^{\prime} & 0 \\
t_{\Delta, \phi_{1}}^{*} & \epsilon_{\mu, k} & 0 & t^{\prime} \\
-t^{\prime} & 0 & -\epsilon_{\mu, k} & t_{\Delta, \phi_{2}} \\
0 & t^{\prime} & t_{\Delta, \phi_{2}}^{*} & \epsilon_{\mu, k}
\end{array}\right]
$$

with $\epsilon_{\mu, k}=(2 t \cos k+\mu)$ and $t_{\Delta, \phi_{j}}=-2 i \Delta \sin k e^{i \phi_{j}} ; j=$ 1,2 and ${ }^{*}$ denotes the complex conjugation. Diagonalizing the Hamiltonian, the dispersion relation is given by

$$
E(k)= \pm \sqrt{\epsilon_{\mu, k}^{2}+t^{\prime 2}+\tau_{\Delta, k} \pm 2 t^{\prime} \sqrt{\epsilon_{\mu, k}^{2}+\tau_{\Delta, k} \sin ^{2} \frac{\Phi}{2}}},
$$

where $\tau_{\Delta, k}=4 \Delta^{2} \sin ^{2} k$ and $\Phi=\phi_{2}-\phi_{1}$. The ladder supports four bands in the band structure due to bonding and antibonding of both holes and electrons.

\section{A. Flat band and localization}

Flat bands are dispersionless bands in the energy spectrum of the translationally invariant lattice Hamiltonian. The Hamiltonian is fine tuned to specific parameter values, leading to strictly flat bands in the entire Brillouin zone. A network could possess one or more flat bands that may be isolated or involve crossings with dispersive bands. In all the known cases, eigenstates corresponding to isolated flat bands are compact localized eigenstates (CLS) which reside on a finite volume of the lattice and absolutely vanishing in the rest of the lattice [17]. In some particular cases, when flat bands make an intersection with a dispersive band, a new type of extended eigenstate usually called as line state exists [42].

The ladder shown in Fig. 1(a) without the $p$-wave pairing term (i.e., $\Delta=0$ ) possesses two dispersive bands. The introduction of the $p$-wave pairing term, along with a careful choice of the parameters, can help to engineer dispersionless bands. There are two main parameter sets that we consider in this study: (1) $\Phi=0, \mu=t^{\prime}, \Delta=t$ and (2) $\Phi=\pi, \mu=$ $0, \Delta=t$. The first parameter set results in a band structure with two dispersive and two flat bands while the second results in four flat bands (Fig. 2). There may be other parameter sets which yield flat bands, and perhaps they may be found with the help of a recently proposed prescription [43]. We point
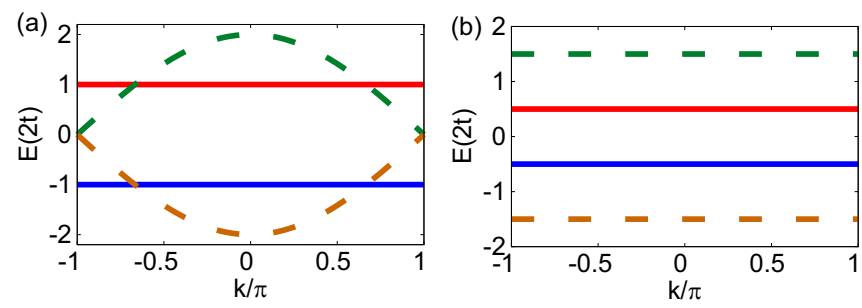

FIG. 2. The dispersion spectrum of system with (a) $\Phi=0, \mu=$ $t^{\prime}=1, \Delta=t=1$, and (b) $\Phi=\pi, \mu=0, \Delta=t=1, t^{\prime}=1$. 
out that the Kitaev chain itself admits flat bands; the flat band condition and the corresponding CLS here are discussed in the Appendix.
To obtain a finer understanding of the flat band conditions, it is useful to rewrite the Hamiltonian in Eq. (4) as

$$
\mathcal{H}(k)=\left[\begin{array}{cc}
-t e^{i k}-t e^{-i k}-\mu & -\Delta e^{i k} e^{i \phi_{1}}+\Delta e^{-i k} e^{i \phi_{1}} \\
\Delta e^{i k} e^{-i \phi_{1}}-\Delta e^{-i k} e^{-i \phi_{1}} & t e^{i k}+t e^{-i k}+\mu \\
-t^{\prime} & 0 \\
0 & t^{\prime}
\end{array}\right.
$$

In this form, the Hamiltonian may be interpreted to represent a new lattice as shown in Fig. 1(b). Equations (4) and (6) are equivalent, with Eq. (6) representing the hopping configurations of the electron and hole explicitly. The new lattice, an interlinked cross-stitch lattice (ICS), consists of a unit cell with four sites which repeats in one spatial direction generating the whole lattice. The mapping leaves the dispersion relation [Eq. (4)] unchanged, resulting in the same four bands in the dispersion diagram. The ICS Hamiltonian also possesses the particle-hole symmetry. The ICS is a modified version of the well-studied cross-stitch lattice, which under certain conditions supports flat bands.

$$
\text { 1. } \Phi=0, \mu=t^{\prime}, \Delta=t
$$

The parameter set results in the dispersion relation $E=$ $\pm 2 t, \pm 2 \sqrt{t^{2}+t^{\prime 2}+2 t t^{\prime} \cos [k]}$ which corresponds to two flat bands and two dispersive bands as shown in Fig. 2(a). Keeping the constraints $\mu=t^{\prime}, \Delta=t$ and varying the parameters, the flat bands can be positioned to cross the dispersive bands or kept isolated from the dispersive bands. This choice of parameters brings forth some interesting topological properties which are discussed in the next section. The CLS corresponding to each flat band can be identified as shown in Fig. 3. The CLS resides on two unit cells, i.e., eight lattice sites, and has strictly zero amplitude outside the two unit cells which is guaranteed by the destructive interference of the probability amplitudes of the CLS at these sites. A translation of relevant unit cell across the length of the lattice results in the other CLS. Thus, the number of CLS for each flat band depends on the system size.

$$
\text { 2. } \Phi=\pi, \mu=0, \Delta=t
$$

This parameter set results in the dispersion relation $E=$ $\pm\left(2 t \pm t^{\prime}\right)$ yielding four flat bands as shown in Fig. 2(b). The eigenstates corresponding to the flat bands are the compact localized states (CLS) which are identified in Fig. 4. This CLS also resides on two unit cells, i.e., eight lattice sites, and has strictly zero amplitude outside the two unit cells as before. Thus, the eigenstate corresponding to the original Kitaev ladder would also be localized and the transport will be prohibited for these parameter sets leaving the ladder in an insulating phase. Furthermore, the condition $t^{\prime}=0$ leads to two uncoupled regular cross-stitch chains, each possessing two flat bands.

$$
\left.\begin{array}{cc}
-t^{\prime} & 0 \\
0 & t^{\prime} \\
-t e^{i k}-t e^{-i k}-\mu & -\Delta e^{i k} e^{i \phi_{2}}+\Delta e^{-i k} e^{i \phi_{2}} \\
\Delta e^{i k} e^{-i \phi_{2}}-\Delta e^{-i k} e^{-i \phi_{2}} & t e^{i k}+t e^{-i k}+\mu
\end{array}\right] .
$$

\section{B. Toplological properties}

One of the most striking features of the Kitaev ladder $[23,44]$ is its topological property. The Kitaev ladder encounters a change of topological class [24,45] from BDI to D $[18,46]$ when subjected to a superconducting phase that breaks time-reversal symmetry. The BDI class Kitaev ladder undergoes a quantum phase transition when the winding number $[18,44]$ changes from two to one to zero in the absence of superconducting phase [41]. The energy spectrum in the absence of superconducting phase difference $\Phi$ is shown in Fig. 5(a). The topological region is of two types. The region $|\mu|<2 t-t^{\prime}$ is characterized by the presence of four zero-energy Majorana edge states with winding number two, while the region $2 t-t^{\prime}<|\mu|<2 t+t^{\prime}$ is characterized by the presence of two zero-energy Majorana edge states with winding number one. The trivial phase with winding number zero lies beyond $|\mu|=2 t+t^{\prime}$ featuring no zero-energy Majorana states. Figure 5(b) shows the energy spectrum when the superconducting phase difference is set to be $\Phi=\pi$. We

(a)

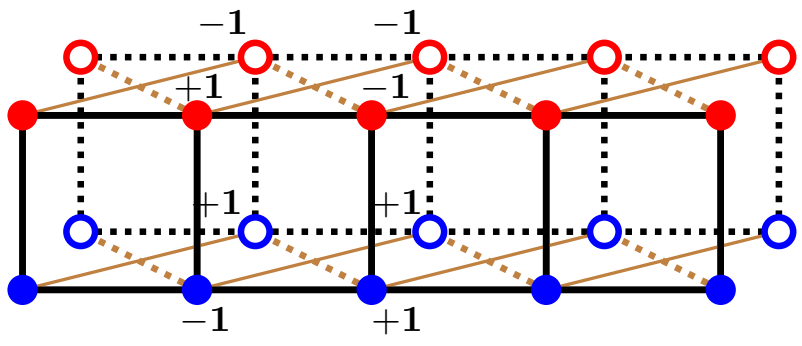

(b)

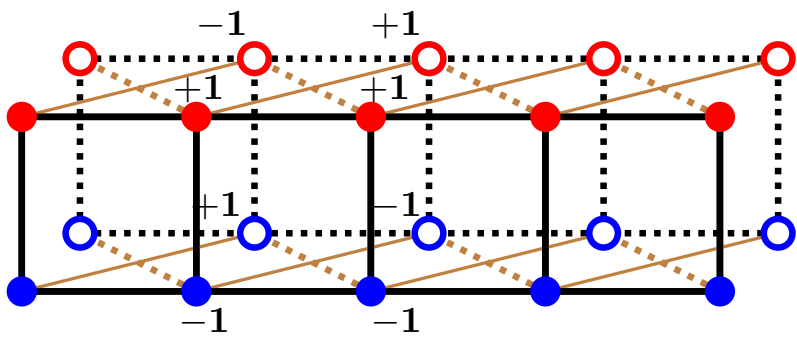

FIG. 3. The compact localized states corresponding to (a) $E=$ -2 , (b) $E=2$ and system parameters are $\Phi=0, \mu=t^{\prime}=1, \Delta=$ $t=1$. The dotted lines represent hopping -1 and regular lines represents hopping +1 . The CLS resides on eight lattice sites with amplitude \pm 1 on those sites. 


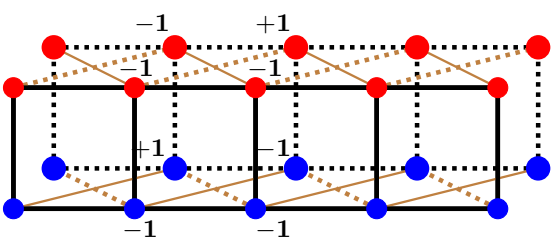

(a)

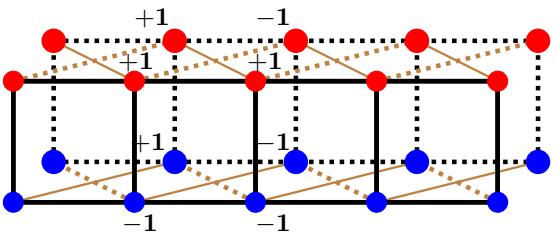

(b)

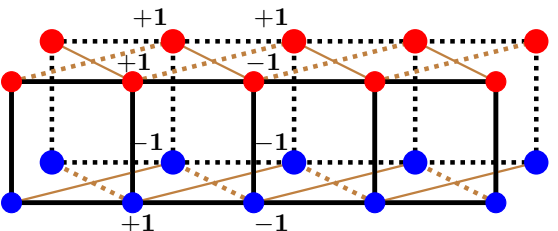

(c)

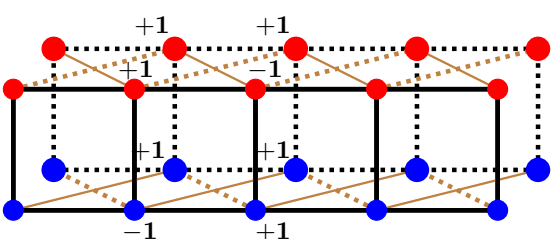

(d)

FIG. 4. The compact localized states corresponding to (a) $E=3$, (b) $E=1$, (c) $E=-1$, (d) $E=-3$ and system parameters are $t=$ $\Delta=1, \mu=0, t^{\prime}=1, \phi_{1}=0, \phi_{2}=\pi$. The dotted lines represent hopping -1 and regular lines represent hopping +1 . The CLS resides on eight lattice sites with amplitude \pm 1 on those sites.

see two distinct twofold-degenerate states at $\pm \frac{t^{\prime}}{t}$ indicating a partial lifting of the fourfold degeneracy of the zero-energy states in Fig. 5(a). Also, the system is now classified into the $\mathrm{D}$ class because of the broken time-reversal symmetry. The topological-to-trivial phase transition is also signaled by the Majorana number $M[24,41]$. We see multiple crossings of energy bands starting from $|\mu|=2 t-t^{\prime}$ to $|\mu|=2 t+t^{\prime}$. These crossings of bands increase the degeneracies of the system and are nicely captured by entanglement entropy as shown in the next section.
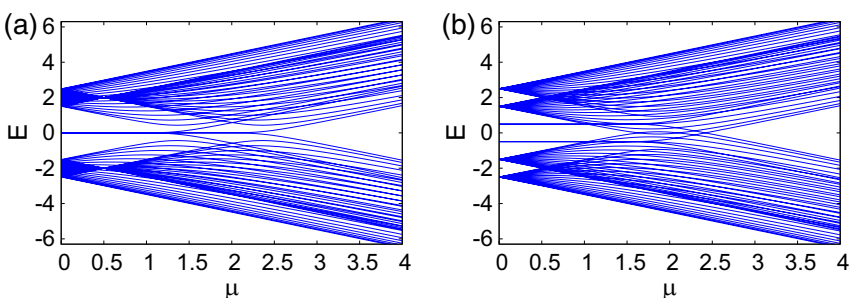

FIG. 5. The single-particle energy spectrum of Kitaev ladder with various onsite chemical potential $\mu$ under imposed open boundary conditions. The other system parameters are chosen to be $L=20$, $t=\Delta=1.0, t^{\prime}=0.5$ with (a) $\Phi=0$, (b) $\Phi=\pi$.

\section{ENTANGLEMENT ENTROPY}

Entanglement $[26,27]$ is one of the most fundamental aspects of many-body quantum systems. It reveals the quantum correlations between the different partitions under study. The most commonly used quantifier of entanglement [47] for a pure state is the bipartite von Neumann entropy: $S_{A}=$ $-\operatorname{Tr}\left(\rho_{A} \ln \rho_{A}\right)$, with $\rho_{A}$ being the reduced density matrix of the subsystem $A$ calculated by tracing out the degrees of freedom of the subsystem $B$. In the noninteracting limit with no pairing term, the entanglement entropy of a subsystem can be effectively calculated with the help of the subsystem correlation matrix: $C_{m n}=\left\langle c_{m}^{\dagger} c_{n}\right\rangle$. It has been shown [27,48,49] that the entanglement entropy is connected to the eigenvalues of this correlation matrix. The correlation matrix approach can be further generalized to study Hamiltonians including a pairing term by considering an additional correlation matrix: $F_{m n}=\left\langle c_{m}^{\dagger} c_{n}^{\dagger}\right\rangle$.

To study this system, we use the well-known LSM method [50] introduced by Lieb, Schultz, and Mattis. Using the LSM formalism, one can write the Hamiltonian in Eq. (1) as the sum of a Hermitian matrix $A$ and a non-Hermitian matrix $B$ as given in Eq. (7):

$$
H=\sum_{i, j=1}^{N} c_{i}^{\dagger} A_{i j} c_{j}+\frac{1}{2} \sum_{i, j=1}^{N}\left(c_{i}^{\dagger} B_{i j} c_{j}^{\dagger}-c_{i} B_{i j}^{*} c_{j}\right),
$$

where $c_{i}$ is a fermionic annhilation operator on the $i$ th site of the leg $a$ for $i=1,2, \ldots, L$ and on the $(i-L)$ th site of the leg $b$ for rest of the values of $i, N=2 L$ being the total number of sites of the ladder system. Upon diagonalization with the help of the Bogoliubov transformation $\eta_{\alpha}=\sum_{i=1}^{N}\left(\frac{1}{2}\left[\Phi_{\alpha}(i)+\Psi_{\alpha}(i)\right] c_{i}+\frac{1}{2}\left[\Phi_{\alpha}(i)-\Psi_{\alpha}(i)\right] c_{i}^{\dagger}\right)$, the Hamiltonian takes the form

$$
H=\sum_{\alpha=1}^{N} \Lambda_{\alpha}\left(\eta_{\alpha}^{\dagger} \eta_{\alpha}-1 / 2\right) \text {. }
$$

The normalized vectors $\Phi_{\alpha}, \Psi_{\alpha}$ and the energy spectrum $\Lambda_{\alpha}$ can be obtained by solving two coupled equations [51] for $\Phi_{\alpha}$ and $\Psi_{\alpha}$ :

$$
\begin{aligned}
& (A-B) \Phi_{\alpha}=\Lambda_{\alpha} \Psi_{\alpha}, \\
& (A+B) \Psi_{\alpha}=\Lambda_{\alpha} \Phi_{\alpha} .
\end{aligned}
$$

In order to compute [52] the entanglement entropy, one has to calculate the correlation matrix of the system. The full system correlation matrix is given by

$$
G_{i j}=\left\langle G S\left|\left(c_{i}^{\dagger}-c_{j}\right)\left(c_{i}^{\dagger}+c_{j}\right)\right| G S\right\rangle,
$$

where $i, j=1,2, \ldots, N$. This correlation matrix can be computed using normalized functions $\Phi_{\alpha}$ and $\Psi_{\alpha}$ as

$$
G_{i j}=-\sum_{\alpha=1}^{N} \Psi_{\alpha}(i) \Phi_{\alpha}(j) .
$$

The anticommutation properties of the $\eta$ operators allow us to obtain the useful relations $\left\langle\eta_{i}^{\dagger} \eta_{j}\right\rangle=\delta_{i j}$ and $\left\langle\eta_{i} \eta_{j}\right\rangle=0$. To compute von Neumann entanglement entropy, one first selectively takes out the part of the full correlation matrix which connects to the subsystem of interest as $G_{s}$. Then, the 

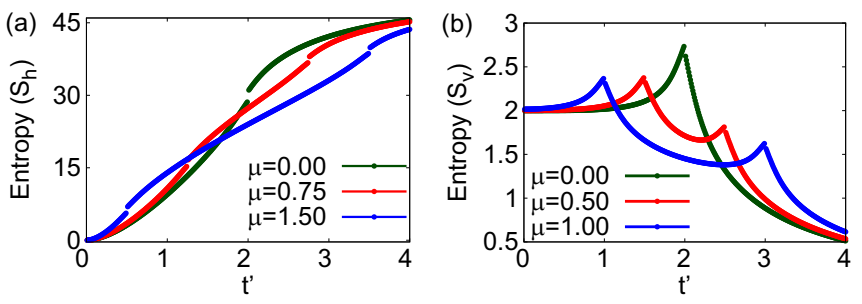

FIG. 6. (a) The entanglement entropy $\left(S_{h}\right)$ of leg $a$ of the Kitaev ladder with respect to the other leg $b$ in units of $\ln 2$. (b) The entanglement entropy $\left(S_{v}\right)$ of half the Kitaev ladder with respect to the other half of the ladder in units of $\ln 2$ for the case of vertical division. The parameters are chosen to be $L=50, t=\Delta=1.0$, $\Phi=0\left(\phi_{1}=\phi_{2}=0\right)$ with various $t^{\prime}$ along with different $\mu$ values under periodic boundary conditions.

entanglement entropy is computed using the method discussed in Ref. [40]. Thus,

$$
S=-\left(\frac{1+v_{\alpha}}{2}\right) \ln \left(\frac{1+v_{\alpha}}{2}\right)-\left(\frac{1-v_{\alpha}}{2}\right) \ln \left(\frac{1-v_{\alpha}}{2}\right),
$$

where $v_{\alpha}$ is the square root of the eigenvalues of the matrix $G_{s} G_{s}^{T}$.

In this work, we analyze two sets of parameters that yield flat bands, and employ entanglement entropy to study the similarities and differences of their properties. Furthermore, in each of these cases, it is useful to consider two types of partitions of the system, which we call horizontal and vertical divisions. The horizontal division makes the two legs of the ladder as two partitions of the system whereas the vertical division considers the two halves of the ladder with ladder length $\frac{L}{2}$ as the two subsystems of interest.

\section{A. Phase difference $\Phi=0$}

In this section, the superconducting phase difference between the two legs of the ladder is maintained at $\Phi=0\left(\phi_{1}=\right.$ $\phi_{2}=0$ ). The dispersion relation of the Kitaev ladder system under this condition is [shown in Fig. 5(a)]

$$
E(k)= \pm \sqrt{\left(2 t \cos k+\mu \pm t^{\prime}\right)^{2}+4 \Delta^{2} \sin ^{2} k} .
$$

The interleg hopping $t^{\prime}$ results in intermixing of two Kitaev chain bands, whose effects are well studied with the help of entanglement entropy. First, the behavior of entanglement entropy for various interleg hopping $t^{\prime}$ of the Kitaev ladder is shown for the two types of partitions in Fig. 6. As depicted in Fig. 6(a) in the absence of interleg hopping $t^{\prime}$, the two legs are disconnected, which results in zero entanglement entropy between both legs. As interleg hopping $t^{\prime}$ is increased, the two legs start to connect through rungs hopping which contributes toward the entanglement entropy between the two legs. The system exhibits two quantum phase transitions at $t^{\prime}=2 t \pm \mu$. We have seen earlier that at these critical values, the band closes, and simultaneously, the winding number undergoes a change from 2 to 1 at $t^{\prime}=2 t-\mu$, and from 1 to 0 at $t^{\prime}=2 t+\mu$ [Fig. 5(a)]. Now, we see from Fig. 6 that the entanglement entropy calculated either via the horizontal or vertical division offers a sharp signature at each of these phase

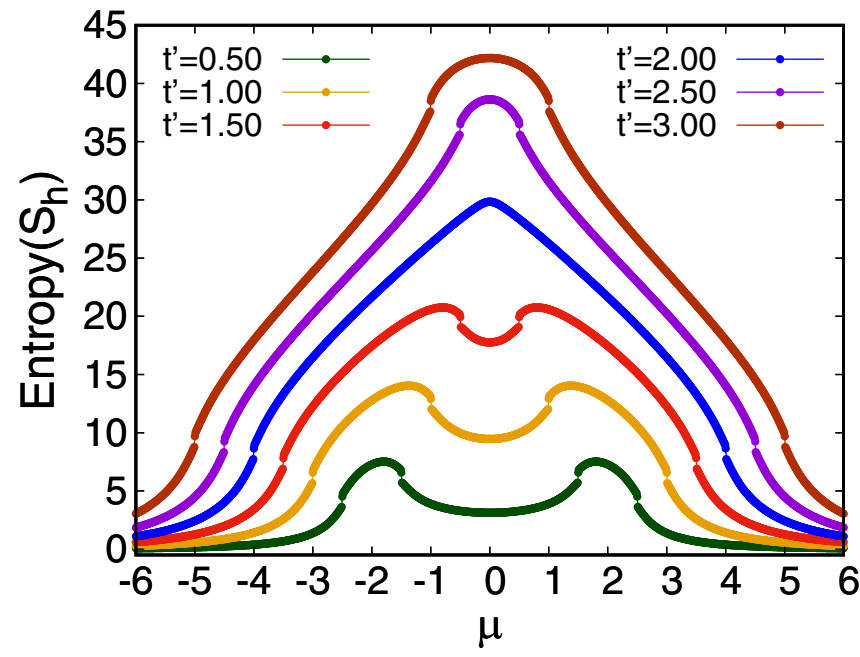

FIG. 7. The entanglement entropy $\left(S_{h}\right)$ in units of $\ln 2$ of Kitaev ladder under horizontal division with various filling $\mu$, for different rungs hopping $t^{\prime}$. The parameters are set to $L=50, t=\Delta=1.0$, $\Phi=0$ and periodic boundary conditions are imposed.

transitions. For strong interleg hopping $t^{\prime}$ the two legs are strongly correlated and the system approaches the maximally entangled state with entanglement entropy $L \ln 2$. One can think of the system tending to a state consisting of $L$ singlets, one over each rung of the ladder. When open boundary conditions are imposed onto the system, degenerate Majorana edge modes also contribute to entanglement entropy $\left(S_{h}\right)$ resulting a starting value of $\ln 2$ rather than zero for small interleg hopping amplitudes $\left(t^{\prime}\right)$.

The same behavior can be seen in Fig. 6(b) when the system is divided vertically. In the absence of interleg hopping $t^{\prime}$ the two legs of the ladder behave as two independent $\mathrm{Ki}$ taev chains each contributing $\ln (2)$ to entanglement entropy. However, strong interleg hopping $t^{\prime}$ increases the quantum correlations between the two legs of the ladder, and weakens the quantum correlations along the legs of the ladder. This causes the vertical division entanglement entropy $S_{v}$ to fall with $t^{\prime}$. The other striking feature from this figure is the occurrence of a kink in $S_{v}$ at the quantum phase transition.

In Fig. 7, the behavior of the horizontal division entanglement entropy $\left(S_{h}\right)$ with filling $\mu$ is shown. For large $|\mu|$ the system tends to approach either the fully filled state or the fully empty state, yielding zero entanglement entropy $\left(S_{h}\right)$ in each case as shown in Fig. 7. A discontinuity in the entropy is seen at the four quantum phase transition points, i.e., $|\mu|=2 t \pm t^{\prime}$. The system possesses two types of quantum phases as described in Fig. 5(a). The entropy features different behavior in different topological regions. The case $t^{\prime}=2 t$ is special, where two of the discontinuities merge at the origin ( $\mu=0$, corresponding to half-filling). We expect a maximum entanglement entropy close to half-filling (i.e., $\mu \sim 0$ ) for $t^{\prime}>2 t$ as confirmed in Fig. 7. In contrast, for $t^{\prime}<2 t$ in the topological region, we see that the entanglement entropy features a broader flat region close to $\mu \sim 0$ due to weakened singlets between the legs of the ladder. This broader region goes to zero for $t^{\prime} \rightarrow 0$ but under open boundary conditions it starts from $\ln 2$ due to the presence of degenerate Majorana edge modes. 


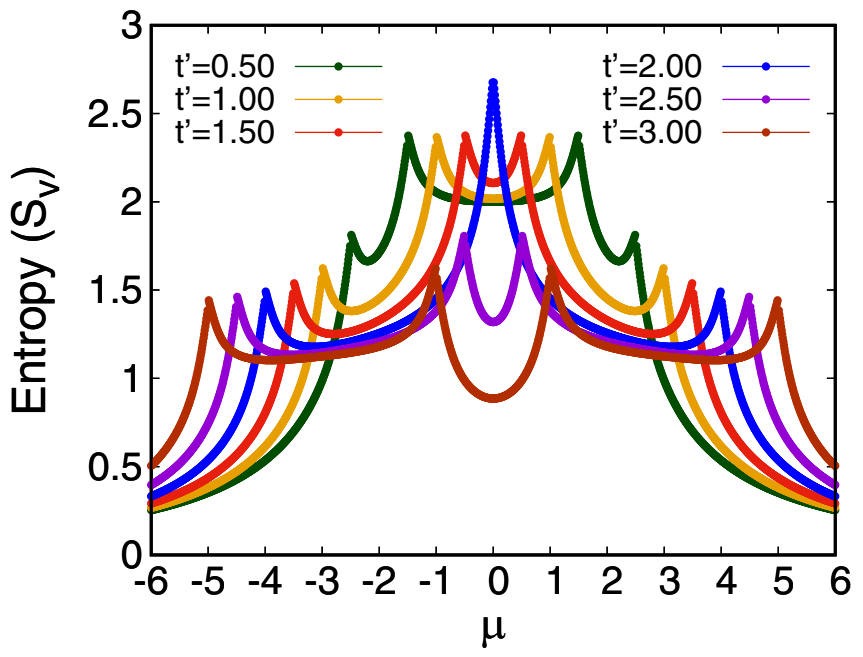

FIG. 8. The entanglement entropy $\left(S_{v}\right)$ in units of $\ln 2$ of Kitaev ladder under vertical division with various filling $\mu$, for different rungs hopping $t^{\prime}$ for set of parameters $L=50, t=\Delta=1.0, \phi=0$ and under imposed periodic boundary conditions.

The vertical division entanglement entropy $\left(S_{v}\right)$ between two halves of the ladder, shown in Fig. 8, is also very revealing. The peaks signal the critical points $(|\mu|=2 t \pm$ $\left.t^{\prime}\right)$ corresponding to quantum phase transitions. The entanglement entropy $\left(S_{v}\right)$ close to half-filling $(\mu \sim 0)$ signifies the change from $2 \ln 2$ to zero when the system switches from a topological to a trivial phase, just like in the normal Kitaev chain. In contrast to $S_{h}$ this entanglement entropy $\left(S_{v}\right)$ is small in the limit of strong interleg hopping $t^{\prime}$ and high in the limit of small $t^{\prime}$ due to formation of strong quantum correlations along the legs of the superconducting ladder.

As we know, the flat band is a special case leading to high degeneracy in the system. In the absence of superconducting phases $\Phi=0$ with $t=\Delta$ and $\mu=t^{\prime}$ [in Eq. (14)], the Kitaev ladder possesses two flat bands and two dispersive bands [Fig. 2(a)]. The behavior of the entanglement entropy under such conditions is shown in Fig. 9 with $\mu=t^{\prime}$. The flat band limit is attained by varying filling in the system, which results in low saturation values of $S_{h}$ as shown in Fig. 9(a). There is only one quantum phase transition point at $t^{\prime}=t$. The discontinuity in entanglement entropy in both Figs. 9(a) and $9(\mathrm{~b})$ is a clear signature of this quantum phase transition at $t^{\prime}=t$.
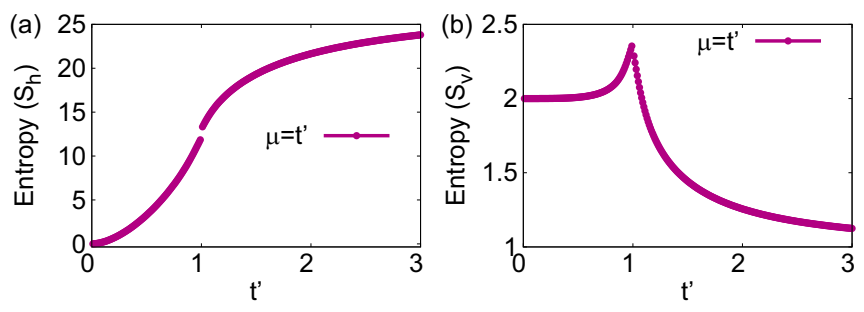

FIG. 9. The flat band entanglement entropy (a) $S_{h}$, (b) $S_{v}$ of the Kitaev ladder with various $t^{\prime}$ for the set of parameters $L=50, t=$ $\Delta=1.0, \phi=0, t^{\prime}=\mu$ in units of $\ln 2$.

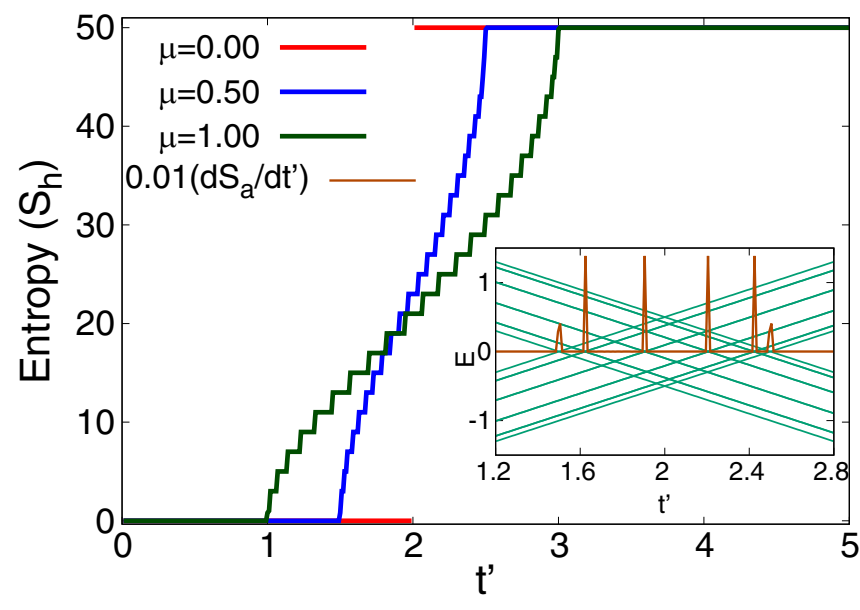

FIG. 10. The entanglement entropy $\left(S_{h}\right)$ of the upper leg of Kitaev ladder with respect to the other leg of the ladder with various $t^{\prime}$ for the parameters $L=50, t=\Delta=1.0, \Phi=\pi\left(\phi_{1}=0, \phi_{2}=\right.$ $\pi$ ) in units of $\ln 2$. The periodic boundary conditions are imposed to the system. The inset represents variation of energy spectrum and the derivative of entanglement entropy $\left(S_{h}\right)$ of Kitaev ladder with the interleg hopping $t^{\prime}$ for the choice of system parameters $L=10$, $t=\Delta=1, \mu=0.5, \Phi=\pi$.

\section{B. Phase difference $\Phi=\pi$}

In this section, the superconducting phase difference is maintained at $\Phi=\pi\left(\phi_{1}=0, \phi_{2}=\pi\right)$. Again, the focus of the study will be the two types of entanglement entropy, i.e., $S_{h}$ and $S_{v}$. The role of the flat band that appears on a further fine tuning of the parameters is then studied as a special case. Figure 10 illustrates the nature of the horizontal division entanglement entropy $\left(S_{h}\right)$ as a function of the interleg hopping $t^{\prime}$.

The dispersion relation for the Kitaev ladder with $\Phi=\pi$ is given by

$$
E(k)= \pm\left(\sqrt{(2 t \cos k+\mu)^{2}+4 \Delta^{2} \sin ^{2} k} \pm t^{\prime}\right) .
$$

So, the Kitaev ladder with $\Phi=\pi$ may be thought of as being effectively made up of two independent Kitaev chains with the origin of the energy shifted by $+t^{\prime}$ in one case and $-t^{\prime}$ in the other. We see from Fig. 10 that the entanglement entropy remains zero, until $t^{\prime}$ hits a threshold value. This feature is to be contrasted with Fig. 6(a) where the entanglement begins to rise for the tiniest of $t^{\prime}$. We can understand this feature as a consequence of the system effectively being two independent chains; entanglement entropy $\left(S_{h}\right)$ remains zero until the energy levels of the two chains cross each other as can be seen from the inset of Fig. 10.

As the coupling strength $t^{\prime}$ is increased, we see a staircaselike increase in the entanglement entropy. We are able to identify the jumps to coincide with a simultaneous crossing of bands in the energy spectrum as shown in the inset of Fig. 10. The point at which bands cross is also a point of degeneracy. Each degenerate state contributes an amount $\ln 2$ to entanglement entropy and so the magnitude of the jump in $S_{h}$ is $\ln 2$ times the degeneracy at the band-crossing point in question. It can be seen that the derivative [53] of entanglement entropy 


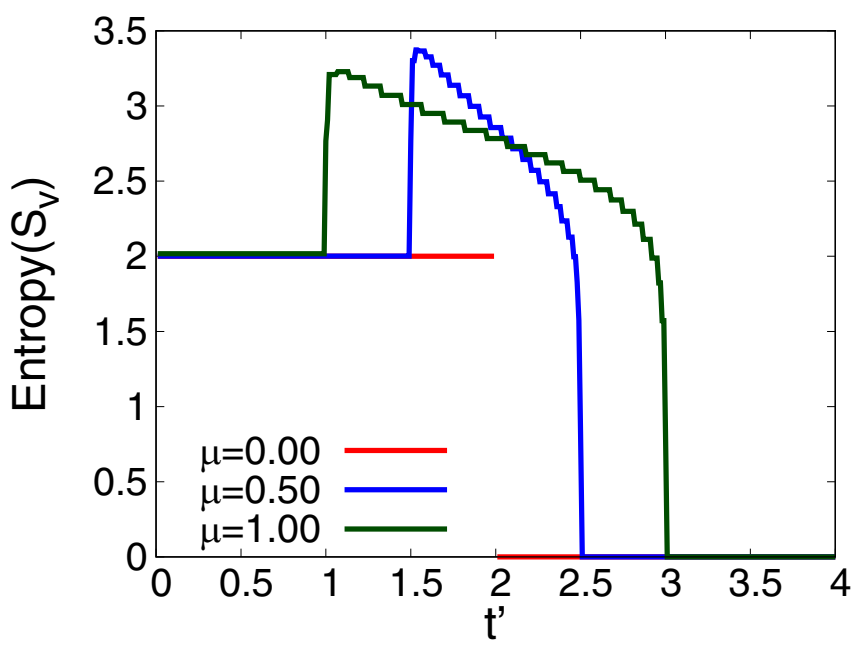

FIG. 11. The entanglement entropy $\left(S_{v}\right)$ in the units of $\ln 2$ of the half Kitaev ladder with respect to the other half of the ladder with various $t^{\prime}$ for set of parameters $L=50, t=\Delta=1.0, \Phi=\pi$.

nicely captures this feature. The staircase structure starts at $t^{\prime}=2 t-\mu$ and saturates at $t^{\prime}=2 t+\mu$. Furthermore, the two critical values are the points where the band closes and the entanglement entropy nicely captures these quantum phase transitions of the system. The entanglement entropy saturates at the maximum possible value of $S_{\text {sat }}=L \ln 2$ where $L$ is the number of rungs of the ladder system. We infer that this is due to the formation of singlets along every rung of the ladder. With open boundary conditions, degenerate Majorana edge modes are present at $\pm \frac{t^{\prime}}{t}$ separately for each of the two independent Kitaev chains of the ladder. These Majorana edge modes again yield higher entanglement entropy $\left(S_{h}\right)$ of $\ln 2$ (as opposed to zero, for periodic boundary conditions) for small interleg hopping amplitudes $\left(t^{\prime}\right)$.

The flat band condition is a special case involving highly degenerate states in the system. It appears when $t=\Delta$, $\mu=0$, in addition to $\Phi=\pi$. For this parameter set, all the four bands are flat, in contrast to the earlier case where two bands were dispersive and two were flat (Fig. 2). It can be seen from Fig. 10 that when the system is tuned to the flat band condition, the horizontal division entanglement entropy $\left(S_{h}\right)$ of the system shows a large and sudden jump from the minimum value, i.e., zero all the way up to the maximum value, i.e., $L \ln 2$ at $t^{\prime}=2 t$. This is due to the crossing of highly degenerate flat energy bands at $t^{\prime}=2 t$. It is worth noting that for the parameter set that we have imposed here, it turns out that each of the constituent Kitaev chains itself is subject to its own flat band condition (i.e., $t=\Delta$ ).

The vertical division entanglement entropy $\left(S_{v}\right)$ confirms the above observations as shown in Fig. 11. Before crossing the lower critical point $t^{\prime}=2 t-\mu$, the entanglement entropy $\left(S_{v}\right)$ is locked at a value of $2 \ln 2$. Once again, this can be understood if we think of the Kitaev ladder as being made of two individual Kitaev chains, each contributing an amount of $\ln 2$ to the entanglement entropy. Also beyond the higher critical point $t^{\prime}=2 t+\mu$, the entanglement entropy becomes zero, confirming the formation of a state made up of singlets along

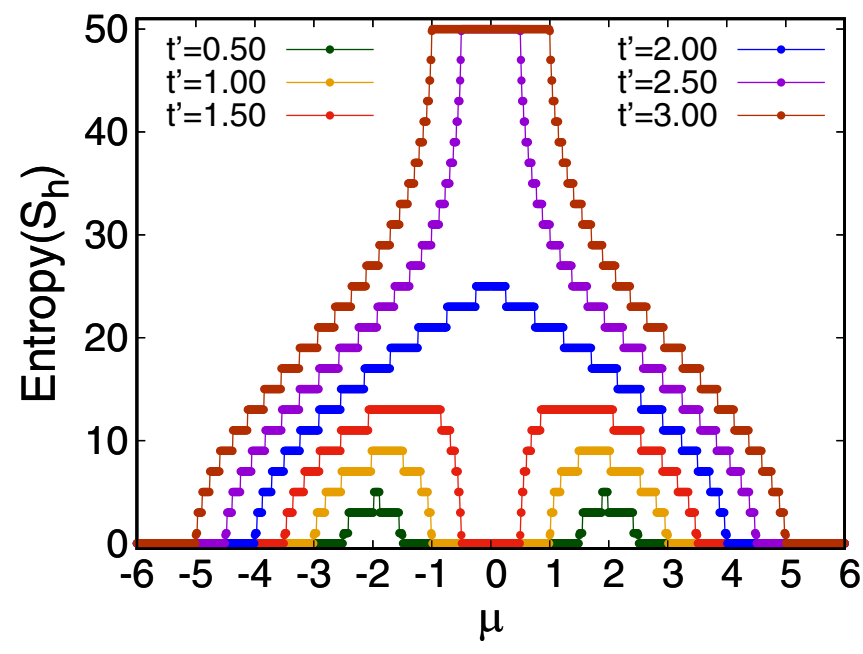

FIG. 12. The horizontal entanglement entropy $\left(S_{h}\right)$ in units of $\ln 2$ of Kitaev ladder with various $\mu$ for parameters $L=50, t=\Delta=$ $1.0, \Phi=\pi$ and for different $t^{\prime}$ values.

each rung of the ladder. In-between these critical bounds, the entanglement entropy shows discontinuous behavior. This is once again understood as a consequence of the crossing of bands at these points. Once again strikingly, when the system is tuned to the flat band condition, there is a single big jump in $S_{v}$ from $2 \ln 2$ to zero at the quantum phase transition.

A study of the dependence of the entanglement entropy as a function of the chemical potential $\mu$ is also instructive. The entanglement pertaining to horizontal division is shown in Fig. 12 while that related to the vertical division is shown in Fig. 13. When the rungs coupling $t^{\prime}$ are very small, the system behaves as two independent Kitaev chains and the topological-to-trivial phase transition occurs at $\mu= \pm(2 t \pm$ $t^{\prime}$ ) as already shown in Fig. 5(b).

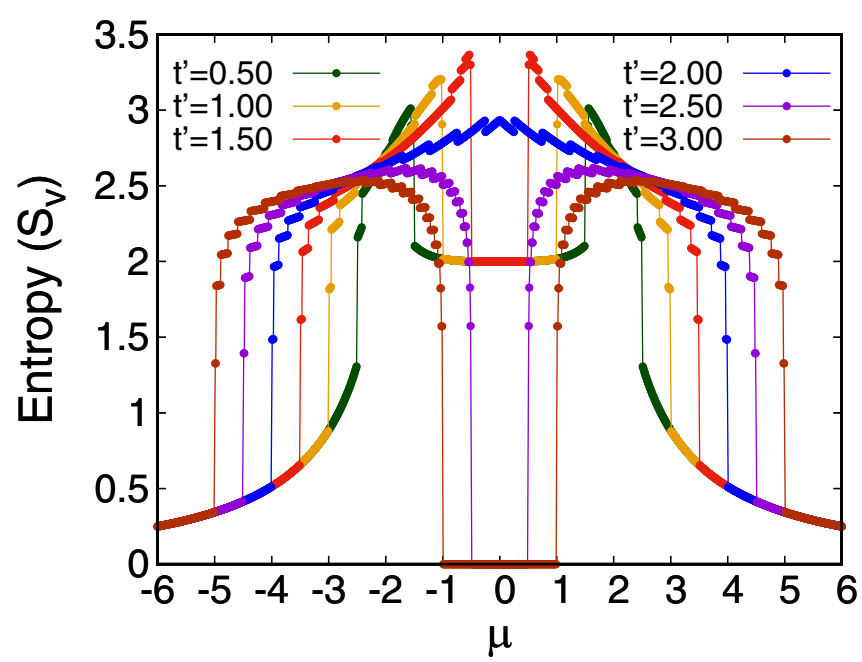

FIG. 13. The entanglement entropy $\left(S_{v}\right)$ of Kitaev ladder under vertical division in units of $\ln 2$ with various filling $\mu$, for different rungs hopping $t^{\prime}$. The parameters are chosen to be $L=50, t=\Delta=$ $1.0, \Phi=\pi$ and periodic boundary conditions are imposed. 
Let us first look at Fig. 12. We see that for any value of $t^{\prime}$, the entanglement entropy $\left(S_{h}\right)$ is a symmetric function of $\mu$ about the origin. We recall from Fig. 5(b) that for $\mu= \pm\left(2 t+t^{\prime}\right)$, the bands cross, and therefore in the regime $|\mu|>\left(2 t+t^{\prime}\right)$ where the two chains behave independently, $S_{h}$ remains zero. In the regime $2 t-t^{\prime}<|\mu|<2 t+t^{\prime}$, we see the entanglement grow in a staircaselike shape, with the jumps happening at points where two bands cross, and whose jump height is given by $\ln 2$ times the degeneracy. We observe that $S_{h}$ first increases and reaches a maximum value which is characteristic of the particular $t^{\prime}$ in question, and has to do with the degree of degeneracy in the dispersion. It then falls again with the overall curve being symmetric in $\mu$. For $\mu=0$, the flat band condition holds (along with $t=\Delta$ and $\Phi=\pi$, which have already been imposed in this section), and we see striking behavior in $S_{h}$ as the system undergoes a transition from the topological to the trivial phase. As we have already seen in Fig. 10, $S_{h}$ undergoes a sudden jump from zero entropy for $t^{\prime}<2 t$ to $L \ln 2$ entropy for $t^{\prime}>2 t$. In Fig. 12, we see that the size of the flat zero entropy region around the origin keeps on shrinking as $t^{\prime}$ approaches $2 t$ from below, and then flips over to a flat maximum entropy region around the origin, whose size increases with $t^{\prime}$ away from $2 t$. However, under open boundary conditions close to half-filling $(\mu=0)$ the entanglement entropy $\left(S_{h}\right)$ features a flip from $\ln 2$ to $L \ln 2$ due to the presence of Majorana edge modes.

Next, we look at the vertical division entanglement entropy $\left(S_{v}\right)$ shown in Fig. 13. Like $S_{h}, S_{v}$ is also symmetric in $\mu$ about the origin. In the regime $|\mu|>\left(2 t+t^{\prime}\right)$ where the two chains behave independently, $S_{v}$ is small but not zero because of weak quantum correlations along the legs of the ladder. At these conditions $|\mu|=2 t+t^{\prime}$ and $|\mu|=2 t-t^{\prime}$, we see jumps in $S_{v}$, like before. We observe that in regions where $S_{h}$ is high, $S_{v}$ tends to be small, presumably because of the underlying monogamy [54] restrictions, which forbids high entanglement of one subsystem with multiple others. The special case of $\mu=0$ corresponding to the flat band condition displays a sharp dramatic change as the system undergoes a topological phase transition. In the topological phase $t^{\prime}<2 t$, $S_{v}=2 \ln 2$, and is zero beyond $t^{\prime}>2 t$, in the trivial phase. We also observe a flat zero entropy region close to $\mu=0$ in the trivial phase, which flips over to a flat region of value $2 \ln 2$ about $\mu=0$ in the topological phase.

\section{SUMMARY}

In this work, we report the existence of flat bands in a Kitaev ladder that includes a $p$-wave superconducting term. These flat bands in dispersion are associated with strongly localized eigenstates known as compact localized states (CLS). With the help of a Bogoliubov transformation, we are able to map the Hamiltonian of the $p$-wave superconductor onto an interlinked cross-stitch lattice, and thus explicitly obtain the underlying compact localized eigenstates.

Studying the dispersion allows us to propose two different parameter sets of the Hamiltonian that yield flat bands. First, we discuss the presence of flat bands for the Kitaev ladder in the absence of superconducting phase difference $\Phi=0$ featuring two dispersive and two flat bands. Next, tuning the superconducting phase difference to $\Phi=\pi$ results in a band structure with only flat bands leaving the system completely insulating. The CLS corresponding to these flat bands are localized on two unit cells of the lattice and with zero amplitude elsewhere. The high localization of these states is responsible for the suppression of transport in the system.

Building on the techniques of Vitagliano et al. [40], we are able to compute many-body entanglement entropy in the eigenstates of the Kitaev ladder. Analogous to numerous other quantum phase transitions, entanglement entropy proves to be a striking diagnostic of the underlying topological-to-trivial phase transition in this system. The inclusion of superconducting phase in the Kitaev ladder gives rise to a change in topological class from BDI to D but entanglement entropy remains a robust diagnostic in both topological classes. For the computation of entanglement entropy, we consider two bipartitions of the system, namely, the horizontal and vertical divisions, which offer complementary insight.

The entanglement entropy is found to strongly depend on the choice of interleg hopping $t^{\prime}$ in both the partitions of the system. Due to underlying monogamy constraints, the trends of the horizontal division entanglement entropy contrast those of the vertical division entanglement entropy. In the absence of superconducting phase difference $(\Phi=0)$, strong interleg hopping connecting the two legs of the ladder tends to enhance the tendency to form singlets along the rungs, and results in high horizontal division entanglement entropy. When the parameters are specially tuned (at $t=\Delta, \mu=t^{\prime}$, and $\Phi=0$ ) to admit a flat band, the horizontal division entanglement entropy seems to be generally suppressed, in particular, featuring lower maximum values compared to non-flat-band tuning. In the presence of superconducting phase difference $(\Phi=\pi)$, the Kitaev ladder can be mapped to two independent Kitaev chains with shifts in energies by $\frac{ \pm t^{\prime}}{t}$. The horizontal division entanglement entropy shows a staircase behavior where the sizes of the steps closely match the degeneracy of the crossing bands. Also, the entanglement entropy features a sharp jump at the flat band condition $(t=\Delta, \mu=0$, and $\Phi=\pi)$ due to the crossing of highly degenerate bands.

We envisage that our work could open up a range of avenues for further exploration. The conventional way to analyze flat bands is to first discover the compact localized states and then to identify the corresponding Hamiltonian. Here, we take the opposite direction; we uncover the existence of flat bands in the very simple Kitaev ladder, followed by detection of corresponding compact localization states. It appears that the $p$-wave pairing symmetry is critical to enabling flat bands in the present system. Our own preliminary calculations (not reported here) indicate that the introduction of the pairing terms along the rungs of the ladder can also yield flat bands. We speculate that this method may be general and may help in engineering other simple geometry lattices that support flat bands. Furthermore, we believe that the study of manybody entanglement entropy could offer fresh insights in other systems. For example, the emergence of Majorana flat bands in $s$-wave and $d$-wave superconductors $[55,56]$ is connected to their underlying topological properties. A study of many-body entanglement entropy in such systems may provide a finer understanding of the topological properties and edge-state behavior of such lattice structures. 


\section{ACKNOWLEDGMENTS}

A.S. is grateful to SERB for the Grant No. (CRG/2019/003447) and DST for the DST-INSPIRE Faculty Award (Grant No. DST/INSPIRE/04/2014/002461). D.S.B. acknowledges Ph.D. fellowship support from UGC India.

\section{APPENDIX: FLAT BANDS IN KITAEV CHAIN}

The Kitaev ladder discussed in this paper has been shown to possess two or four flat bands according to the parameter set chosen for the Hamiltonian. Further, the Kitaev ladder has been mapped on to an interlinked cross-stitch lattice and the eigenstates corresponding to flat bands have been shown as CLS. In this Appendix, we show that setting the rungs hopping $t^{\prime}=0$ separates the Kitaev ladder into two Kitaev chains and the latter can be mapped into a well-known flat band lattice known as cross-stitch lattice.

When $t^{\prime}$ is set to zero, the tight-binding Hamiltonian for Kitaev ladder leads to that for a Kitaev chain and can be represented as

$$
\begin{aligned}
H= & -t \sum_{n} c_{n+1}^{\dagger} c_{n}-\mu \sum_{n} c_{n}^{\dagger} c_{n} \\
& -\Delta \sum_{n} c_{n+1}^{\dagger} c_{n}^{\dagger}+\text { H.c. },
\end{aligned}
$$

where $t$ is the intraleg hopping amplitude, $c_{n}^{\dagger}\left(c_{n}\right)$ are creation (annihilation) operators on the $n$th site of the chain. Through a Bogoliubov transformation [25,41], the overall Hamiltonian in $k$ space is given as

$$
\mathcal{H}(k)=\left[\begin{array}{cc}
-\epsilon_{\mu, k} & t_{\Delta} \\
t_{\Delta}^{*} & \epsilon_{\mu, k}
\end{array}\right]
$$

with $\epsilon_{\mu, k}=(2 t \cos k+\mu)$ and $t_{\Delta}=-2 i \Delta \sin k$. The dispersion relation corresponding to the Kitaev chain can be obtained as

$$
E(k)= \pm \sqrt{\epsilon_{\mu, k}^{2}+\tau_{\Delta, k}}
$$

with $\epsilon_{\mu, k}=(2 t \cos k+\mu)$ and $\tau_{\Delta, k}=4 \Delta^{2} \sin ^{2} k$. The band structure consists of two bands which reflect the particle-hole symmetry of the Hamiltonian. (a)

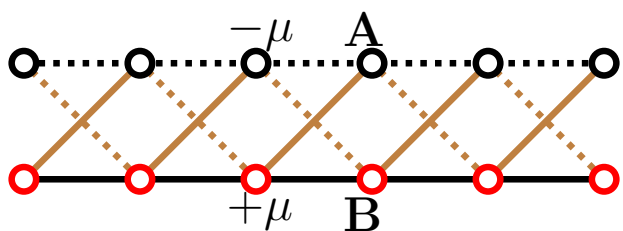

(b)

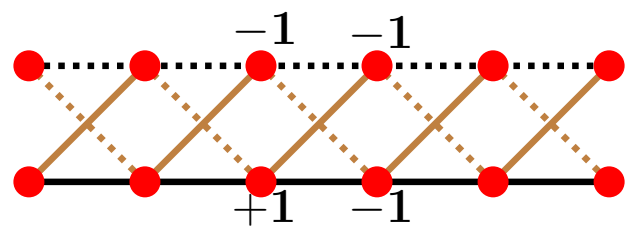

(c)

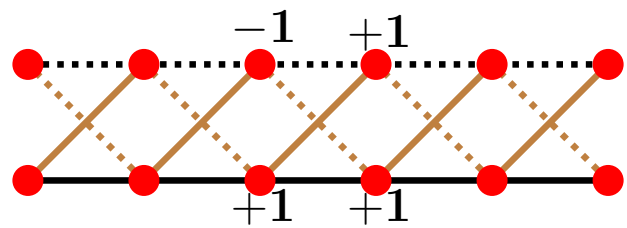

FIG. 14. (a) Schematic representation of the cross-stitch chain. The black (red) open circles represent negative (positive) onsite energies at sites and red dots represents $\mu=0$. The dotted (regular) lines represent negative (positive) hopping. (b), (c) The eigenstates corresponding to $E=-2$ and +2 , respectively, for $\mu=0, t=\Delta=1$.

To understand the flat band properties of the Kitaev chain better, the Hamiltonian can be rearranged as

$$
H=\left(\begin{array}{cc}
-\mu-e^{-i k} t-e^{i k} t & \Delta e^{-i k}-\Delta e^{i k} \\
\Delta e^{i k}-\Delta e^{-i k} & \mu+e^{-i k} t+e^{i k} t
\end{array}\right) .
$$

The resulting Hamiltonian can be mapped onto a cross-stitch chain as shown in Fig. 14(a). The cross-stitch chain consists of two sites per unit cell represented by $A$ and $B$ in the figure. For $\mu=0, t=\Delta=1$, the Kitaev chain and hence the cross-stitch chain support two flat bands at $E= \pm 2$. The CLS corresponding to both flat bands in the cross-stitch chain are shown in Figs. 14(b) and 14(c). The CLS for both bands reside on two unit cells (i.e., four sites) with strictly zero amplitudes in the rest of the chain.
[1] S. A. Parameswaran, R. Roy, and S. L. Sondhi, Fractional quantum hall physics in topological flat bands, C. R. Phys. 14, 816 (2013).

[2] S. Flach, D. Leykam, J. D. Bodyfelt, P. Matthies, and A. S. Desyatnikov, Detangling flat bands into fano lattices, Europhys. Lett. 105, 30001 (2014).

[3] D. Leykam, A. Andreanov, and S. Flach, Artificial flat band systems: From lattice models to experiments, Adv. Phys. 3, 1473052 (2018).

[4] N. W. Ashcroft and N. D. Mermin, Solid State Physics (Saunders College, Philadelphia, 1976), Appendix N.

[5] A. Mielke, Ferromagnetism in the hubbard model on line graphs and further considerations, J. Phys. A: Math. Gen. 24, 3311 (1991).
[6] H. Tasaki, Ferromagnetism in the Hubbard Models with Degenerate Single-Electron Ground States, Phys. Rev. Lett. 69, 1608 (1992).

[7] A. Mielke, Ferromagnetism in Single-Band Hubbard Models with a Partially Flat Band, Phys. Rev. Lett. 82, 4312 (1999).

[8] H. Tasaki, From Nagaoka's ferromagnetism to flat-band ferromagnetism and beyond: An introduction to ferromagnetism in the hubbard model, Prog. Theor. Phys. 99, 489 (1998).

[9] D. Guzmán-Silva, C. Mejía-Cortés, M. Bandres, M. C. Rechtsman, S. Weimann, S. Nolte, M. Segev, A. Szameit, and R. Vicencio, Experimental observation of bulk and edge transport in photonic lieb lattices, New J. Phys. 16, 063061 (2014). 
[10] R. A. Vicencio, C. Cantillano, L. Morales-Inostroza, B. Real, C. Mejía-Cortés, S. Weimann, A. Szameit, and M. I. Molina, Observation of Localized States in Lieb Photonic Lattices, Phys. Rev. Lett. 114, 245503 (2015).

[11] S. Mukherjee, A. Spracklen, D. Choudhury, N. Goldman, P. Öhberg, E. Andersson, and R. R. Thomson, Observation of a Localized Flat-Band State in a Photonic Lieb Lattice, Phys. Rev. Lett. 114, 245504 (2015).

[12] Y. Zhang and C. Zhang, Bose-einstein condensates in spinorbit-coupled optical lattices: Flat bands and superfluidity, Phys. Rev. A 87, 023611 (2013).

[13] S. Gladchenko, D. Olaya, E. Dupont-Ferrier, B. Douçot, L. B. Ioffe, and M. E. Gershenson, Superconducting nanocircuits for topologically protected qubits, Nat. Phys. 5, 48 (2009).

[14] W. Maimaiti, A. Andreanov, H. C. Park, O. Gendelman, and S. Flach, Compact localized states and flat-band generators in one dimension, Phys. Rev. B 95, 115135 (2017).

[15] A. Ramachandran, A. Andreanov, and S. Flach, Chiral flat bands: Existence, engineering, and stability, Phys. Rev. B 96, 161104(R) (2017).

[16] L. Morales-Inostroza and R. A. Vicencio, Simple method to construct flat-band lattices, Phys. Rev. A 94, 043831 (2016).

[17] W. Maimaiti, S. Flach, and A. Andreanov, Universal d = 1 flat band generator from compact localized states, Phys. Rev. B 99, 125129 (2019).

[18] A. Maiellaro, F. Romeo, and R. Citro, Topological phase diagram of a Kitaev ladder, Eur. Phys. J.: Spec. Top. 227, 1397 (2018).

[19] H.-H. Hung, J. Wu, K. Sun, and C.-K. Chiu, Engineering of many-body majorana states in a topological insulator/s-wave superconductor heterostructure, Sci. Rep. 7, 1 (2017).

[20] M. Cheng and H.-H. Tu, Majorana edge states in interacting two-chain ladders of fermions, Phys. Rev. B 84, 094503 (2011).

[21] H. Wang, L. Shao, Y. Pan, R. Shen, L. Sheng, and D. Xing, Flux-driven quantum phase transitions in two-leg kitaev ladder topological superconductor systems, Phys. Lett. A 380, 3936 (2016).

[22] W. DeGottardi, D. Sen, and S. Vishveshwara, Topological phases, majorana modes and quench dynamics in a spin ladder system, New J. Phys. 13, 065028 (2011).

[23] A. Y. Kitaev, Unpaired majorana fermions in quantum wires, Phys.-Usp. 44, 131 (2001).

[24] N. Wu, Topological phases of the two-leg kitaev ladder, Phys. Lett. A 376, 3530 (2012).

[25] R. Nehra, D. S. Bhakuni, A. Sharma, and A. Soori, Enhancement of crossed andreev reflection in a kitaev ladder connected to normal metal leads, J. Phys.: Condens. Matter 31, 345304 (2019).

[26] S. T. Flammia, A. Hamma, T. L. Hughes, and X.-G. Wen, Topological Entanglement Rényi Entropy and Reduced Density Matrix Structure, Phys. Rev. Lett. 103, 261601 (2009).

[27] R. Nehra, D. S. Bhakuni, S. Gangadharaiah, and A. Sharma, Many-body entanglement in a topological chiral ladder, Phys. Rev. B 98, 045120 (2018).

[28] X. Chen and E. Fradkin, Quantum entanglement and thermal reduced density matrices in fermion and spin systems on ladders, J. Stat. Mech.: Theory Exp. (2013) P08013.

[29] J. Eisert, M. Cramer, and M. B. Plenio, Colloquium: Area laws for the entanglement entropy, Rev. Mod. Phys. 82, 277 (2010).
[30] A. Kitaev and J. Preskill, Topological Entanglement Entropy, Phys. Rev. Lett. 96, 110404 (2006).

[31] D. Vodola, L. Lepori, E. Ercolessi, A. V. Gorshkov, and G. Pupillo, Kitaev Chains with Long-Range Pairing, Phys. Rev. Lett. 113, 156402 (2014).

[32] D.-P. Liu, Topological phase boundary in a generalized kitaev model, Chin. Phys. B 25, 057101 (2016).

[33] D. Hügel and B. Paredes, Chiral ladders and the edges of quantum hall insulators, Phys. Rev. A 89, 023619 (2014).

[34] N. Roy and A. Sharma, Entanglement contour perspective for "strong area-law violation" in a disordered long-range hopping model, Phys. Rev. B 97, 125116 (2018).

[35] N. Roy and A. Sharma, Study of counterintuitive transport properties in the aubry-andré-harper model via entanglement entropy and persistent current, Phys. Rev. B 100, 195143 (2019).

[36] A. Dey, D. S. Bhakuni, B. K. Agarwalla, and A. Sharma, Quantum entanglement and transport in non-equilibrium interacting double-dot setup: The curious role of degeneracy, J. Phys.: Condens. Matter 32, 075603 (2020).

[37] A. Sharma and E. Rabani, Landauer current and mutual information, Phys. Rev. B 91, 085121 (2015).

[38] H. S. Sable, D. S. Bhakuni, and A. Sharma, Landauer current and mutual information in a bosonic quantum dot, J. Phys.: Conf. Ser. 964, 012007 (2018).

[39] D. S. Bhakuni and A. Sharma, Characteristic length scales from entanglement dynamics in electric-field-driven tight-binding chains, Phys. Rev. B 98, 045408 (2018).

[40] G. Vitagliano, A. Riera, and J. I. Latorre, Volume-law scaling for the entanglement entropy in spin- $1 / 2$ chains, New J. Phys. 12, 113049 (2010).

[41] R. Nehra, A. Sharma, and A. Soori, Transport in a long-range kitaev ladder: Role of majorana and subgap andreev states, arXiv:1909.00565.

[42] S. Xia, A. Ramachandran, S. Xia, D. Li, X. Liu, L. Tang, Y. Hu, D. Song, J. Xu, D. Leykam et al., Unconventional Flatband Line States in Photonic Lieb Lattices, Phys. Rev. Lett. 121, 263902 (2018).

[43] L. Toikka and A. Andreanov, Necessary and sufficient conditions for flat bands in m-dimensional n-band lattices with complex-valued nearest-neighbour hopping, J. Phys. A: Math. Theor. 52, 02LT04 (2018).

[44] A. Alecce and L. Dell' Anna, Extended kitaev chain with longerrange hopping and pairing, Phys. Rev. B 95, 195160 (2017).

[45] A. Kitaev, Periodic table for topological insulators and superconductors, in Advances in Theoretical Physics: Landau Memorial Conference, edited by V. Lebedev and M. Feigel'man, AIP Conf. Proc. No. 1134 (AIP, Melville, NY, 2009), pp. 22-30.

[46] H. Zhou and J. Y. Lee, Periodic table for topological bands with non-hermitian symmetries, Phys. Rev. B 99, 235112 (2019).

[47] I. Peschel and V. Eisler, Reduced density matrices and entanglement entropy in free lattice models, J. Phys. A: Math. Theor. 42, 504003 (2009).

[48] I. Peschel, Calculation of reduced density matrices from correlation functions, J. Phys. A: Math. Gen. 36, L205 (2003).

[49] I. Peschel, Special review: Entanglement in solvable manyparticle models, Braz. J. Phys. 42, 267 (2012).

[50] E. Lieb, T. Schultz, and D. Mattis, Two soluble models of an antiferromagnetic chain, Ann. Phys. 16, 407 (1961). 
[51] I. Mahyaeh and E. Ardonne, Zero modes of the kitaev chain with phase-gradients and longer range couplings, J. Phys. Commun. 2, 045010 (2018).

[52] S. Vijay and L. Fu, Entanglement spectrum of a random partition: Connection with the localization transition, Phys. Rev. B 91, 220101(R) (2015).

[53] J. Borchmann and T. Pereg-Barnea, Analytic expression for the entanglement entropy of a two-dimensional topological superconductor, Phys. Rev. B 95, 075152 (2017).
[54] D. Yang, A simple proof of monogamy of entanglement, Phys. Lett. A 360, 249 (2006).

[55] S. Deng, G. Ortiz, A. Poudel, and L. Viola, Majorana flat bands in $s$-wave gapless topological superconductors, Phys. Rev. B 89, 140507(R) (2014).

[56] P. Kumar, T. I. Vanhala, and P. Törmä, Magnetization, $d$-wave superconductivity, and non-fermi-liquid behavior in a crossover from dispersive to flat bands, Phys. Rev. B 100, 125141 (2019). 